\title{
Endometrioid adenocarcinoma arising from abdominal wall endometriosis in the appendectomy scar: A case report
}

\author{
Masaru Hayashi $^{1,2}$, Akiko Shoda ${ }^{1}$, Nobuaki Kosaka ${ }^{1}$, Masaru Kojima ${ }^{3}$, Nobuhide Masawa ${ }^{3}$, \\ Ichio Fukasawa ${ }^{1}$ \\ ${ }^{1}$ Department of Obstetrics and Gynecology, Dokkyo Medical University, Tochigi, Japan \\ ${ }^{2}$ Department of Obstetrics and Gynecology, Teine Keijinkai Hospital, Sapporo, Japan \\ ${ }^{3}$ Anatomic and Diagnostic Pathology, Dokkyo Medical University, Tochigi, Japan \\ Email: masaru_hayashi@hotmail.com \\ Received 14 July 2013; revised 13 August 2013; accepted 20 August 2013 \\ Copyright (C) 2013 Masaru Hayashi et al. This is an open access article distributed under the Creative Commons Attribution License, \\ which permits unrestricted use, distribution, and reproduction in any medium, provided the original work is properly cited.
}

\begin{abstract}
We report a very rare case of endometrioid adenocarcinoma arising from abdominal wall endometriosis in the appendectomy scar. A 47-year-old woman visited the surgical department, since she had a gradually growing and painful tumor both in an appendectomy scar and at an umbilical site. She underwent appendectomy at age 18 years, and noticed the tumor at age 22 years. Partial tumor resection was performed in that department, and the pathology revealed endometrioid adenocarcinoma. She was referred to our department for radical therapy. Tumors in the both sites were dissected together with some swelling lymph nodes in our department. A pathological diagnosis of the tumor in the umbilical site showed only benign endometriosis. In contrast, the tumor in the appendectomy scar showed benign endometriosis, atypical endometriosis and well differentiated endometrioid adenocarcinoma. Resected lymph nodes also contained endometrioid adenocarcinoma, and were diagnosed as metastases. It was concluded that the endometrioid adenocarcinoma in the tumor of the appendectomy scar was a malignant transformation arising from abdominal wall endometriosis from the pathological findings. Since the operation, adjuvant and maintenance chemotherapy with paclitaxel and carboplatin had been administered for 3 years. She is free of disease 3.5 years after the operation.
\end{abstract}

Keywords: Abdominal Wall Endometriosis; Appendectomy Scar; Malignant Transformation; Endometrioid Adenocarcinoma

\section{INTRODUCTION}

Endometriosis is a very common gynecological disease in women of reproductive age. Its prevalence is estimated as $10 \%-15 \%$ for them. Although the most common sites of endometriosis are the ovary and the peritoneum in the pelvic cavity, it can also occur in other rare sites, such as the urinary tract, colon, diaphragm, lung, and abdominal wall.

Endometriosis has a potential to undergo malignant transformation. The incidence of malignant transformation in ovarian endometriosis is reported to be $0.7 \%$ $1.0 \%$, and endometriosis in other rare sites may also become malignant. We report a very rare case of endometrioid adenocarcinoma arising from abdominal wall endometriosis in the appendectomy scar.

\section{CASE REPORT}

A 47-year-old woman, gravida 2, para 2 (normal vaginal deliveries), visited the surgical department in anotherhospital because she had gradually growing and painful tumors both in an appendectomy scar and in the umbilicus. She underwent appendectomy at age 18 years, and noticed the tumors at age 22 years. Subcutaneous and superficial bleeding in both tumor sites began during her menstrual periods 8 years ago. Partial tumor resection was performed in the previous hospital, and the pathology of the tumor revealed endometrioid adenocarcinoma. She was referred to our department for radical therapy. When she came to our department, she was administered 3 courses of gonadotropin-releasing hormone analog.

A physical examination and trans-abdominal ultrasonography revealed a $5 \times 3 \mathrm{~cm}$ solid tumor in the appendectomy scar and a $2 \times 1.5 \mathrm{~cm}$ solid tumor in the 
umbilicus. FDG-PET ( ${ }^{18}$ F-fluorodeoxyglucose positron emission tomography) with CT (computed tomography) showed FDG accumulation in both tumors, and also in the right superficial inguinal lymph node. The SUV (Standardized Uptake Value) max was 4.24 by the tumor in the appendectomy scar, 2.56 by the tumor in the umbilical site, and 2.46 in the right inguinal lymph node. There were no other accumulations of FDG. CT showed that both tumors were present mainly in the subcutaneous tissue. However, the tumor in the appendectomy scar had enhanced intensively by a contrast material (Figure 1(a)), and invaded to the abdominal rectus muscle and external oblique muscle of the abdomen (Figure 1(b)). The serum tumor markers CA125 and CA19-9 were slightly elevated to $37 \mathrm{U} / \mathrm{ml}$ and $96 \mathrm{U} / \mathrm{ml}$, respectively. Other tumor markers, such as CEA and SCC were within normal limits. Other laboratory data were also within normal limits.

Macroscopic findings during the operation revealed that the tumor in the appendectomy scar penetrated the abdominal wall, and was partly exposed to the abdominal cavity. The uterus was slightly enlarged because of adenomyosis. There were some blueberry spots on the right ovary and fallopian tube. Left external iliac, obturator, external/internal supra inguinal, and right superficial inguinal lymph nodes were swelled by palpation. Based on these findings, total tumor resection at both sites, total hysterectomy, bilateral salpingo-oophorectomy and dissection of the swelled lymph nodes were performed for an optimal cytoreductive surgery. The tumor in the appendectomy scar was widely dissected together with penetrated and surrounding abdominal wall tissue. The tumor in the umbilical site was well circumscribed and simply resected. On gross macroscopic examination, a 5 $\mathrm{cm}, 38 \mathrm{gr}$ solid pink-gray colored tumor in the umbilical site had blueberry spots and was focally hemorrhagic. A $10 \mathrm{~cm}, 173 \mathrm{gr}$ pink colored solid tumor in the appendectomy scar had two sites of $1 \mathrm{~cm}$ chocolate cysts and some blueberry spots, and were also focally hemorrhagic (Figure 2(a)).

A histological examination showed that the tumor inthe umbilical site was a benign endometriosis with pro-

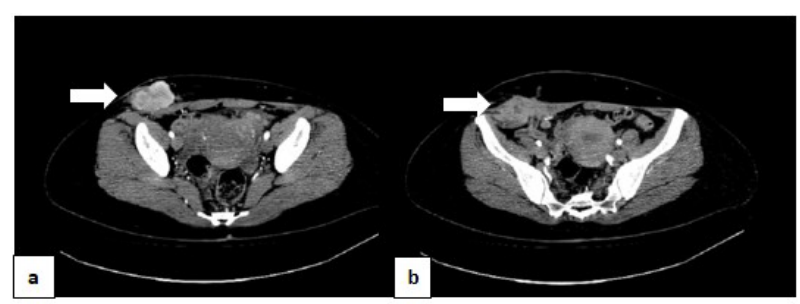

Figure 1. CT showed that the tumor in the appendectomy scar were present mainly in the subcutaneous tissue. However, this tumor had enhanced intensively by a contrast material (arrow) (a); and invaded to the abdominal rectus muscle and external oblique muscle of the abdomen (arrow) (b).
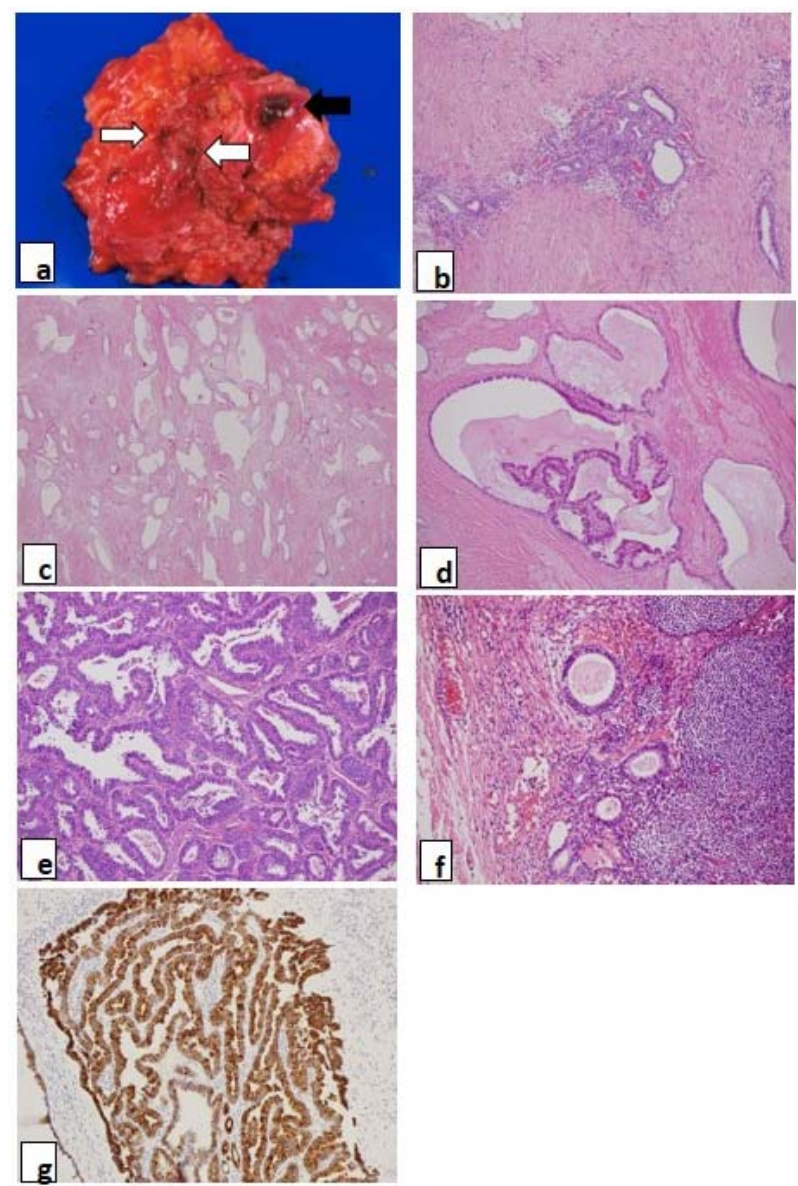

Figure 2. (a) Gross appearance of the tumor in the appendectomy site. Open arrow shows blue-berry spots, and closed arrow shows chocolate cyst in the tumor; (b) The tumor in the umbilical site showed only benign endometriosis, which consisted of endometrial gland and endometrial stroma (H\&E, $\times 200$ ); (c)-(e) The tumor in the appendectomy scar; (c) Benign endometriosis: many endometrial glands proliferated with a little endometrial stroma. Some glands showed complex structures without any cellular atypia (H\&E, $\times 100)$; (d) Atypical endometriosis: this atypical endometriosis showed serial changes from normal epithelium to atypical epithelium in the same endometrial gland (H\&E, ×200); (e) Endometrioid adenocarcinoma: well-differentiated endometrioid adenocarcinoma proliferated (H\&E, ×400); (f) Adjacent lymph nodes to the tumor in the appendectomy scar had only benign endometriosis; (g) CK7 immunoreactivity is shown in the cytoplasm of the carcinoma in the appendectomy scar $(\mathrm{H} \& \mathrm{E}, \times 200)$.

liferated fibrous tissue (Figure 2(b)). The tumor in the appendectomy scar showed benign endometriosis (Figure 2(c)), atypical endometriosis (Figure 2(d)) and welldifferentiated endometrioid adenocarcinoma (Figure 2(e)) together with proliferated fibrous tissue. Most of the benign endometriosis in the appendectomy scar was composed of proliferating endometrial glands, which were complex structures with very little endometrial stroma, as compared with the tumor in the umbilical site. Some of the atypical endometriosis showed serial changes from 
normal epithelium to atypical epithelium in the same endometrial gland (Figure 2(d)). The uterus showed only benign adenomyosis. The ovaries showed benign endometriosis and atypical endometriosis, but not malignancy. All resected lymph node showed endometrioid adenocarcinoma, and diagnosed as metastases. Adjacent lymph nodes to the tumor in the appendectomy scar were coincidently resected. They had not endometrioid adenocarcinoma but benign endometriosis (Figure 2(f)). Table 1 shows a summary of these pathological findings. An immunohistochemical study was performed for ER, PgR, CA125, CK7, CD10, p53, aromatase, CK20 and Ki-67. $p 53$, aromatase, Ki-67 and CK20 were negative at all sites. CK7 was strongly positive for all epithelium of the glands in benign, atypical endometriosis and endometrioid adenocarcinoma (Figure 2(g)). CA125 was positive to strongly positive in benign endometriosis of all sites, and atypical endometriosis in the ovary. CD10 was strongly positive for the endometrial stromal cells of benign endometriosis of the tumor in the umbilical site, but showed various staining patterns for the endometrial stromal cells of benign and atypical endometriosis at other sites. ER and PgR were positive to strongly positive in benign endometriosis of the tumor in the umbilical site and also for that of atypical endometriosis in the ovary. But ER was negative, and PgR was only weakly positive for benign endometriosis of the tumor in the appendectomy scar. ER, PgR, CA125 and CD10 were all negative for atypical endometriosis of the tumor in the appendectomy scar and endometrial adenocarcinoma of all sites. Table 2 shows a summary of these immunohistochemical studies.

We concluded that this endometrioid adenocarcinoma in the appendectomy scar arose from the abdominal wall endometriosis by these pathological findings. Since the operation, 6 courses of adjuvant chemotherapy and 10 courses of maintenance chemotherapy with paclitaxel and carboplatin had been administered for 3 years. She is free of disease 3 and half years after the operation.

Table 1. Summary of pathology.

\begin{tabular}{cccc}
\hline & $\begin{array}{c}\text { Benign } \\
\text { endometriosis }\end{array}$ & $\begin{array}{c}\text { Atypical } \\
\text { endometriosis }\end{array}$ & Adenocarcinoma \\
\hline $\begin{array}{c}\text { Tumor in the } \\
\text { appendectomy scar } \\
\text { Tumor in the } \\
\text { umbilical site }\end{array}$ & ++ & + & +++ \\
Ovary & ++ & - & - \\
Lymph nodes & + & + & - \\
Uterus & + & - & $+++{ }^{* *}$ \\
\hline
\end{tabular}

- negative, + positive, ++ strongly positive, +++ extremely positive. ('Lymph nodes: Adjacent lymph nodes to the tumor in the appendectomy scar, ${ }^{* *}$ Lymph nodes: Left external iliac, obturator, external/internal supra inguinal, and right superficial inguinal lymph nodes).
Table 2. Summary of immunohistochemical study.

\begin{tabular}{|c|c|c|c|c|c|}
\hline & ER & PgR & CA125 & CK7 & CD10 \\
\hline $\begin{array}{l}\text { Benign endometriosis } \\
\text { of the tumor } \\
\text { in the umbilical site }\end{array}$ & ++ & ++ & ++ & ++ & ++ \\
\hline $\begin{array}{l}\text { Benign endometriosis } \\
\text { in the ovary }\end{array}$ & ++ & ++ & ++ & ++ & + \\
\hline $\begin{array}{l}\text { Atypical endometriosis } \\
\text { in the ovary }\end{array}$ & + & ++ & + & ++ & $-\sim+$ \\
\hline $\begin{array}{l}\text { Benign endometriosis } \\
\text { of the tumor } \\
\text { in the appendectomy scar }\end{array}$ & - & \pm & $+\sim++$ & ++ & $-\sim+$ \\
\hline $\begin{array}{l}\text { Atypical endometriosis } \\
\text { of the tumor } \\
\text { in the appendectomy scar }\end{array}$ & - & - & - & ++ & - \\
\hline $\begin{array}{c}\text { Endometrioid } \\
\text { adenocarcinoma } \\
\text { in the appendectomy scar }\end{array}$ & - & - & - & ++ & - \\
\hline $\begin{array}{l}\text { Endometrioid } \\
\text { adenocarcinoma } \\
\text { in lymph nodes }\end{array}$ & - & - & - & ++ & - \\
\hline
\end{tabular}

${ }^{* *}$ All were negative for $p 53$, aromatase, CK20, Ki-67-negative staining, \pm weakly positive staining, + positive staining, ++ strongly positive.

\section{DISCUSSION}

Abdominal wall endometriosis is defined as any ectopic endometrial tissue superficial to peritoneum, and usually associated with a cesarean section scar. Horton J.D. et al. reviewed 445 cases of abdominal wall endometriosis [1] and reported that $55 \%$ of cases were associated with a cesarean section scar, $11 \%$ with hysterectomy, $13 \%$ with other types of surgical scar, and $20 \%$ were not associated with an operation scar. The incidence of abdominal wall endometriosis after cesarean section was estimated to be from $0.03 \%$ to $1 \%$. The mean age was 31.4 years, $96 \%$ presented with a mass, $87 \%$ presented with pain, and $57 \%$ presented with cyclic symptoms. Cutaneous endometriosis without any surgical history are referred to as spontaneous abdominal wall endometriosis. The most common sites of this endometriosis are reported at the umbilicus and the groin [2].

In the pathogenesis of abdominal wall endometriosis, implantation of endometrial tissue by a uterus-related surgery or retrograde menstruation is widely accepted. The other hypotheses such as vascular (lymphatic and hematogenous) metastasis of endometrial tissue, and coelomic metaplasia of endometriosis are also proposed. Spontaneous abdominal wall endometriosis is frequently explained by vascular metastasis theory. Jafferbhoy S. hypothesizes that scars seem to have a tendency to attract endometrial tissue and the umbilicus behaves as a physiological scar. That is why abdominal scars and the umbilicus show a predilection to metastasis for endometrial tissue [3]. Our case does not have any past histories about uterus-related surgery. Moreover, she clearly re- 
membered that it was not during menstruation at the time when the appendectomy was undergone. In this case, the hypothesis of vascular metastasis is supposed to be adapted. Because the adjacent the lymph nodes to the tumor in the appendectomy scar contained endometriosis, and this case also had umbilical endometriosis.

Although $75 \%$ of malignant transformation of endometriosis occurs in the ovary, $25 \%$ of the cases occur in extra-ovary [4]. Ulrich U. et al. [5] reported that the most frequent site in 139 cases of extra-ovarian cancer arising from endometriosis was the bowel (28\%), followed by the rectovaginal septum (13\%), uterus (arising from adenomyosis) (12\%), peritoneum (6\%) and others (such as broad ligament, parametrium, urinary bladder, and vagina) (44\%). Reviews on the histology of malignant transformation in extra-ovarian endometriosis are primarily represented by endometrioid adenocarcinoma (69.1\%), followed by sarcoma (25\%) and clear cell carcinoma (4.5\%) [6].

Malignant transformation of abdominal wall endometriosis is extremely rare. Only 28 cases have been reported so far [7-10]. In the histology of this malignancy, clear cell adenocarcinoma comprised 64\%, followed by $14 \%$ of endometrioid adenocarcinoma, $7 \%$ of papillary serous adenocarcinoma, and others. All 28 cases had a past history of abdominal surgery. In terms of the kind of previous surgery, 82\% were cesarean sections and the others were uterus-related operations (hysterectomy, myomectomy, laparotomy for uterine perforation) or endometrial cysts of the ovary. Only our case involved nonobstetrical/gynecological surgery.

Three histological criteria for malignant transformation arising from endometriosis were proposed by Sampson in 1925: 1) demonstration of both benign and neoplastic endometrial tissue in the tumor, 2) histological type of tumor compatible with endometrial origin, and 3) no other primary tumor site [11]. Contrasting these criteria with our case, 1) we demonstrated both benign endometriosis and carcinoma in the tumor of the appendectomy scar. 2) In addition to the morphology showing a well-differentiated endometrioid adenocarcinoma based on $\mathrm{H} \& \mathrm{E}$ staining, a CK7 positive/CK20 negative staining pattern was highly suggestive of an endometrial gland origin [12,13]. 3) There was no clinical evidence of other primary sites.

A fourth criterion was proposed by Scott in 1953, who added the concept of a transitional zone that existed as a dysplastic phase between benign endometriosis and carcinoma [14]. However, Benoit L. et al. reported in his review [4] that the transitional zone was only detected in $36 \%-46 \%$ of malignant extra-ovarian endometriosis. In Mostoufizadeh's opinion, coexistence of a neoplasm and benign endometrial tissue is sufficient to demonstrate the endometrial origin of the lesion [15]. In the 23 reported cases of malignant transformation of abdominal wall endometriosis, only 13 cases were presented with macroscopic or microscopic evidence of coexisting endometriosis [7]. We could not find a transitional zone in our case, but we found benign endometriosis, endometrioid adenocarcinoma as well as atypical endometriosis in the tumor of the appendectomy scar. Atypical endometriosis is supposed to be closely associated with the development of endometrioid adenocarcinoma in malignant transformation of ovarian endometriosis $[16,17]$. Some of the atypical endometriosis in the tumor of the appendectomy scar showed serial changes from normal epithelium to atypical epithelium in the same endometrial gland. The benign endometriosis in the tumor of the appendectomy scar showed no cellular atypia of the epithelium, but showed an atypical glandular structure. In the immunohistochemical study, benign endometriosis in the tumor of the appendectomy scar showed a close similarity to both atypical endometriosis and endometrioid adenocarcinoma in the same site, as compared with benign endometriosis at other sites. Taken together, benign endometriosis, atypical endometriosis and endometrioid adenocarcinoma in the tumor of the appendectomy scar were closely related and supposed to be serial change. Therefore, we concluded that this endometrioid adenocarcinoma arose from the abdominal wall endometriosis.

Because of the rarity of malignant transformation of abdominal wall endometriosis, a standard treatment is difficult to establish. In the review of 28 cases, the basic treatment was radical resection. In some cases, additional surgery was performed, such as total hysterectomy, bilateral salpingo-oophorectomy, pelvic lymphadenectomy, and omentectomy. Moreover, in some cases, adjuvant chemotherapy, radiotherapy, or progesterone therapy were selected. However, the efficacy of these treatments is unclear. In terms of the prognosis of 25 of the 28 cases (3 cases had no data about it), 8 cases died from the disease, 13 cases had no evidence of disease, and 4 case recurred during the follow-up period from 6 weeks to 60 month [7-10]. According to our standard treatment in advanced ovarian cancer, we chose 6 courses of adjuvant chemotherapy after the operation followed by 10 courses of maintenance chemotherapy every 3 months with paclitaxel and carboplatin for 3 years, because cancer occurred beyond the primary site, including multiple lymph node metastases. Optimal cytoreductive surgery and this chemotherapy is very effective because the patients free of disease 3 and half years after the operation by clinical examination including FDG-PET with CT.

\section{REFERENCES}

[1] Horton, J.D., DeZee, K.J., Ahnfeldt, E.P. and Wagner, M. (2008) Abdominal wall endometriosis: A surgeon's perspective and review of 445 cases. The American Journal of Surgery, 196, 207-212. 


\section{doi:10.1016/j.amjsurg.2007.07.035}

[2] Efremidou, E.I., Kouklakis, G., Mitrakas, A., Liratzopoulos, N. and Polychronidis, A.C. (2012) Primary umbilical endometrioma: A rare case of spontaneous abdominal wall endometriosis. International Journal of General Medicine, 5, 999-1002. doi:10.2147/IJGM.S37302

[3] Jafferbhoy, S., Symeonides, P., Levy, M. and Shiwani, M.H. (2013) Chronic umbilical discharge: An unusual presentation of endometriosis. Sultan Qaboos University Medical Sciences Journal, 13, 143-146.

[4] Benoit, L., Arnould, L., Cheynel, N., Diane, B., Causeret, S., Machado, A., Collin, F., Fraisse, J. and Cuisenier, J. (2006) Malignant extraovarian endometriosis: A review. European Journal of Surgical Oncology, 32, 6-11. doi:10.1016/j.ejso.2005.08.011

[5] Ulrich, U., Rhiem, K., Kaminski, M., Wardelmann, E., Trog, D., Valter, M. and Richter, O.N. (2005) Parametrial and rectovaginal adenocarcinoma arising from endometriosis. International Journal of Gynecological Cancer, 15, 1206-1209. doi:10.1111/j.1525-1438.2005.00184.X

[6] Bats, A.S., Zafrani, Y., Pautier, P., Duvillard, P. and Morice, P. (2008) Malignant transformation of abdominal wall endometriosis to clear cell carcinoma: Case report and review of the literature. Fertility and Sterility, 90, 1197.e13-1197.e16. doi:10.1016/j.fertnstert.2007.08.080

[7] Li, X., Yang, J., Cao, D., Lang, J., Chen, J. and Shen, K. (2012) Clear-cell carcinoma of the abdominal wall after cesarean delivery. Obstetrics \& Gynecology, 120, 445448. doi:10.1097/AOG.0b013e31824da6fe

[8] Shalin, S.C., Haws, A.L., Carter, D.G. and Zarrin-Khameh, N. (2012) Clear cell adenocarcinoma arising from endometriosis in abdominal wall cesarean section scar: A case report and review of the literature. Journal of Cutaneous Pathology, 39, 1035-1041. doi:10.1111/j.1600-0560.2012.01982.x
[9] Sawazaki, H., Goto, H., Takao, N., Taki, Y. and Takeuchi, H. (2012) Clear cell adenocarcinoma arising from abdominal wall endometriosis mimicking urachal tumor. Urology, 79, 84-85. doi:10.1016/j.urology.2011.02.034

[10] Mert, I., Semaan, A., Kim, S., Ali-Fehmi, R. and Morris, R.T. (2012) Clear cell carcinoma arising in the abdominal wall: Two case reports and literature review. American Journal of Obstetrics \& Gynecology, 207, 7-9. doi:10.1016/j.ajog.2012.05.029

[11] Sampson, J.A. (1925) Endometrial carcinoma of the ovary, arising in endometrial tissue in that organ. Archives of Surgery, 10, 1-2. doi:10.1001/archsurg.1925.01120100007001

[12] Wang, N.P., Zee, S., Zarbo, R.J., Baccchi, C.E., Gown, A.M. (1995) Coordinate expression of cytokeratins 7 and 20 defines unique subsets of carcinomas. Applied Immunohistochemistry \& Molecular Morphology, 3, 99-107.

[13] Chu, P., Wu, E. and Weiss, L.M. (2000) Cytokeratin 7 and cytokeratin 20 expression in epithelial neoplasms: A survey of 435 cases. Modern Pathology, 13, 962-972. doi:10.1038/modpathol.3880175

[14] Scott, R. (1953) Malignant changes in endometriosis. Obstetrics \& Gynecology, 2, 283-289.

[15] Mostoufizadeh, M. and Scully, R.E. (1980) Malignant tumors arising in endometriosis. Clinical Obstetrics and Gynecology, 23, 951-963.

[16] Fukunaga, M., Nomura, K., Ishikawa, E. and Ushigome, S. (1997) Ovarian atypical endometriosis: Its close association with malignant epithelial tumours. Histopathology, 30, 249-255. doi:10.1046/j.1365-2559.1997.d01-592.x

[17] Terada, T. (2012) Endometrioid adenocarcinoma of the ovary arising in atypical endometriosis. International Journal of Clinical and Experimental Pathology, 5, 924927. 